PAPER • OPEN ACCESS

The frustration of being odd: how boundary conditions can destroy local order

To cite this article: Vanja Mari et al 2020 New J. Phys. 22083024

View the article online for updates and enhancements. 
New Journal of Physics

The open access journal at the forefront of physics
Published in partnership with: Deutsche Physikalische Gesellschaft and the Institute of Physics

\title{
PAPER
}

CrossMark

OPEN ACCESS

RECEIVED

18 March 2020

REVISED

4 June 2020

ACCEPTED FOR PUBLICATION

26 June 2020

PUBLISHED

11 August 2020

Original content from

this work may be used

under the terms of the

Creative Commons

Attribution 4.0 licence.

Any further distribution

of this work must

maintain attribution to

the author(s) and the

title of the work, journal

citation and DOI.

\section{The frustration of being odd: how boundary conditions can destroy local order}

\author{
Vanja Marićc ${ }^{1,2}$, Salvatore Marco Giampaolo ${ }^{1}$, Domagoj Kuić ${ }^{1}$ and Fabio Franchini ${ }^{1,}$ \\ 1 Division of Theoretical Physics, Ruđer Bošković Institute, Bijenička cesta 54, 10000 Zagreb, Croatia \\ 2 SISSA and INFN, via Bonomea 265, 34136 Trieste, Italy \\ 3 Author to whom any correspondence should be addressed. \\ E-mail: fabio@irb.hr \\ Keywords: order parameter, frustrated magnetism, antiferromagnetism, spin chains, thermodynamic limit \\ Supplementary material for this article is available online
}

\begin{abstract}
A central tenant in the classification of phases is that boundary conditions cannot affect the bulk properties of a system. In this work, we show striking, yet puzzling, evidence of a clear violation of this assumption. We use the prototypical example of an XYZ chain with no external field in a ring geometry with an odd number of sites and both ferromagnetic and antiferromagnetic interactions. In such a setting, even at finite sizes, we are able to calculate directly the spontaneous magnetizations that are traditionally used as order parameters to characterize the system's phases. When ferromagnetic interactions dominate, we recover magnetizations that in the thermodynamic limit lose any knowledge about the boundary conditions and are in complete agreement with standard expectations. On the contrary, when the system is governed by antiferromagnetic interactions, the magnetizations decay algebraically to zero with the system size and are not staggered, despite the antiferromagnetic coupling. We term this behavior ferromagnetic mesoscopic magnetization. Hence, in the antiferromagnetic regime, our results show an unexpected dependence of a local, one-spin expectation values on the boundary conditions, which is in contrast with predictions from the general theory.
\end{abstract}

\section{Introduction}

Landau theory is one of the most impactful constructions of the last century. It allows distinguishing between different phases through different local order parameters, quantities which are finite or vanish depending on the phase of a system [1-4]. Although the new century has taught us that this classification is not complete, because certain phases of quantum matter are characterized by non-local order (for instance, topological [5-13]), Landau theory remains a cornerstone to interpret phases, directly borrowed from classical statistical mechanics.

Order parameters are supposed to capture macroscopic properties of phases and thus are believed not to depend on boundary conditions. Indeed, as the boundary contributions are typically sub-extensive, they should bring a negligible effect for sufficiently large systems. Of course, depending on the system and on the type of interactions, there can be ambiguities on what 'sufficiently large system' means, as sometimes boundary effects can decay just algebraically, even in phases considered gapped [14, 15]. Thus, the standard prescription to characterize a phase is to take the thermodynamic limit before evaluating physical observables $[1,3]$.

This being said, the effects of boundary conditions have been a subject of interest in different contexts. For instance, the Kondo effect can largely be interpreted as a boundary effect [16, 17]. But additional simple examples that have received a lot of attention immediately come to mind, such as conformal field theories (CFTs) and integrable models. In the former, conformal invariance poses tight bounds on the bulk properties and it has been established that boundary condition modifies 
the system's equilibrium behavior [18-20]. In the latter, different boundary conditions are commonly employed to study various properties. For instance, the partition functions of $2 \mathrm{D}$ classical systems with domain wall boundary conditions provide the normalization of the corresponding quantum wave-functions [21]. But certain boundary conditions are also known to generate the phenomenon of the 'arctic curve', which separate frozen regions (due to boundary conditions) from liquid ones [22-30].

A particularly thorny issue is represented by frustration [31, 32]. This term evokes different phenomena to different ears. While it simply refers to the presence of interactions promoting incompatible orderings (hence the impossibility of simultaneously minimizing every term in the system's Hamiltonian [33-35]), the effects of frustration are varied and complex [36-38]. Frustrated systems are a debated and very active field of research, with a rich phenomenology (different in many ways from that of non-frustrated systems) and with unique challenges [37]. Nonetheless, at the heart of every frustrated system one can find one (or typically many) frustrated loops, which are the building blocks out of which the different phenomenologies arise [39]. Here, we will concentrate on this simplest, and original, incarnation of geometrical frustration. This is a classical concept which applies, for instance, to Ising spins coupled anti-ferromagnetically. While, locally, there is no problem in satisfying the antiferromagnetic (AFM) interactions, when the spins are arranged in a loop made of an odd number of sites, at least one bond needs to display ferromagnetic alignment. In this case, the frustration arises because of an incompatibility between local interactions and the global structure of the system and is due to the particular choice of boundary conditions (namely, periodic with an odd number of sites, which we term 'frustrated boundary conditions', FBC). It is worth to note that, while for loops with an even number of sites the lowest energy state is doubly degenerate (given by the two types of Neel orders), with frustration the degeneracy becomes extensive because the defect can be placed on any bond of the ring. Hence, by adding a single site to a ring, the problem turns from a non-degenerate perturbative one to a problem of degenerate perturbation theory, which is a priori non-trivial due to the extensive degeneracy in the thermodynamic limit.

Upon adding quantum interactions to a geometrically frustrated system, we can generally expect the degeneracy to be lifted. A perturbative approach characterizes the resulting ground state as the superposition of a delocalized excitation on top of the non frustrated ground state. This picture was recently checked in [15] for its validity even beyond the perturbative regime and confirmed using the entanglement entropy. The effects of such delocalized excitation have been studied in the past [40-42], revealing subtle phenomena, usually dismissed by the community as peculiar quirks, because these analyses never addressed local observables, but rather properties such as the spectral gap.

In this work, we pluck a hole in this canvas by focusing instead on the order parameter of AFM spin chains and by showing that FBC makes it vanish as the ring's length diverges. To the best of our knowledge, this is the first example of a case in which boundary conditions affect local observables (in thermodynamic limit) and it is in evident contrast with standard general arguments recapped above. Nonetheless, we should remark that evidence pointing toward a vanishing of the spontaneous magnetization with FBC was already reported, for instance through the two-point function $[14,15]$, but in previous works, the importance of finite-size effects was harder to estimate. We should also stress that our result is consistent with the single-particle picture mentioned above, as the traveling excitation destroys local order by flipping every spin in its motion. At the moment, we do not know how to reconcile our findings with the traditional paradigm, although we can speculate that, being geometrical frustration a non-local effect, some sort of topological mechanism is at play so that we propose to call the interplay between quantum interaction and FBC with the term 'topological frustration'. Our evidence is inescapable and should compel the community to understand what makes the spin chains we consider different and so sensitive to the boundary conditions, so to understand what class of models share the same or similar behaviors and how do they fit in the standard paradigm.

After introducing the systems under consideration (namely, a class of spin- $\frac{1}{2}$ chains with a global $\mathbb{Z}_{2}$ symmetry), we will recap the two complementary approaches to extract the order parameter in the symmetry broken phase in absence of frustration and then apply the same techniques to the case with FBC. Doing so, we benchmark our technique, showing that it yields the expected results in the former case, while it shows that frustration suppresses the order parameter to zero. In our work, we consider both models where we can perform exact analytical calculations to prove our claims and generalizations in which we have to resort to numerical diagonalization. We will show that for non-frustrated systems the order parameter grows to saturation exponentially with growing total system size, while it decreases toward zero algebraically with FBC. 


\section{The spin chains and their properties}

We consider an anisotropic spin- $\frac{1}{2}$ chain with Hamiltonian

$$
H=\sum_{j=1}^{N} \cos \delta\left(\cos \phi \sigma_{j}^{x} \sigma_{j+1}^{x}+\sin \phi \sigma_{j}^{y} \sigma_{j+1}^{y}\right)-\sin \delta \sigma_{j}^{z} \sigma_{j+1}^{z},
$$

where $\sigma_{j}^{\alpha}$, with $\alpha=x, y, z$, are Pauli operators and $N$ is the number of lattice sites, which we henceforth set to be odd $N=2 M+1$. Crucially, we apply periodic boundary conditions $\sigma_{j+N}^{\alpha}=\sigma_{j}^{\alpha}$.

The model is expected to exhibit a quantum phase transition every time two of the couplings are, in magnitude, equal and greater than the third [43] (in that case, the model becomes equivalent to a critical XXZ chain [44]). Dualities are connecting different rearrangements of the couplings along the $x, y$, and $z$ directions [43]. Moreover, to avoid additional effects (and degeneracies) that will be the subject of subsequent works [45], we will allow only one AFM coupling (namely, along the $x$ direction), letting the other two to favor a ferromagnetic alignment. We thus limit the range of the anisotropy parameters such that $\phi \in[-\pi / 2,0]$ and $\delta \in[0, \pi / 2]$, so that the phase transition is at $\phi=-\pi / 4$ (for $\tan \delta<1 / \sqrt{2}$ ) and separates two phases characterized by a two-fold degenerate ground state. In particular, for $\phi \in[-\pi / 2,-\pi / 4)$ the phase favors a ferromagnetic alignment along the $y$ direction $(y \mathrm{FM})$, while for $\phi \in(-\pi / 4,0]$ the dominant interaction is AFM along the $x$ direction (xAFM) and thus topologically frustrated.

With no external field, all three parity operators along the three axes $\Pi^{\alpha} \equiv \bigotimes_{j=1}^{N} \sigma_{j}^{\alpha}$ commute with the XYZ Hamiltonian in equation $(1)\left(\left[H, \Pi^{\alpha}\right]\right)$. Moreover, since we are considering systems made by an odd number of sites $N=2 M+1$, the $\Pi^{\alpha}$ do not commute with one another, but rather anti-commute $\left\{\Pi^{\alpha}, \Pi^{\beta}\right\}=2 \delta_{\alpha, \beta}$ and actually fulfill a SU(2) algebra. This structure implies that every state is exactly degenerate an even number of times, also on a finite chain. If $|\Psi\rangle$ is an eigenstate, say, of $\Pi^{z}$, then $\Pi^{x}|\Psi\rangle$, that differs from $\Pi^{y}|\Psi\rangle$ by a global phase factor, is also an eigenstate of the Hamiltonian with opposite $z$-parity but with the same energy.

Applying an external magnetic field $h$ along, say, the $z$-direction leaves only $\Pi^{z}$ to commute with the Hamiltonian, thus restoring the original $\mathbb{Z}_{2}$ symmetry the model is known for and breaking the exact finite-size degeneracy between the states $[44,46]$. Nonetheless, up to a critical value of $h$, it is known that the induced energy split is exponentially small in the system size [47] and thus that the degeneracy is restored in the thermodynamic limit, representing one of the simplest, and most cited, examples of spontaneous symmetry breaking (SSB) [46]. To simplify things, let us set $\delta=0$, so that equation (1) describes an anisotropic XY chain [44, 48]. For $|h|<1$ we are in the SSB phase. This means that, although a ground state with definite $z$-parities necessarily has zero expectation value concerning $\sigma_{j}^{x}$ and $\sigma_{j}^{y}$, in the thermodynamic limit the degeneracy allows to select a ground state which is a superposition of different $z$-parities, which can thus display a spontaneous magnetization in the $x$ or $y$ direction. In the $y$ FM phase we expect the order parameter $m_{y} \equiv\left\langle\sigma_{j}^{y}\right\rangle$ to be finite, while in the $x$ AFM the non-vanishing order parameter should be the staggered magnetization $m_{x} \equiv(-1)^{j}\left\langle\sigma_{j}^{x}\right\rangle$.

\section{The ferromagnetic case}

Let us now turn back to the system in equation (1) and focus on the ferromagnetic region $\phi \in[-\pi / 2,-\pi / 4)$. The (quasi-)long-range order represented by the order parameter can be extracted in two ways: either from the two-point function or by selecting a suitable superposition of states at finite sizes and then following their magnetization toward the thermodynamic limit. While the second is easily amenable to numerical approaches, the former, which is most suitable for analytical techniques, takes advantage of the cluster decomposition property

$$
\lim _{r \rightarrow \infty}\left\langle\sigma_{j}^{\alpha} \sigma_{j+r}^{\alpha}\right\rangle-\left\langle\sigma_{j}^{\alpha}\right\rangle\left\langle\sigma_{j+r}^{\alpha}\right\rangle=0,
$$

to extract the order parameter from the large distance behavior of the system's two-point correlators.

Exploiting the Jordan-Wigner transformation [44, 49], which maps the spin degrees of freedom into spin-less fermions:

$$
c_{j}=\left(\bigotimes_{l=1}^{j-1} \sigma_{l}^{z}\right) \frac{\sigma_{j}^{x}+\imath \sigma_{j}^{y}}{2}, \quad c_{j}^{\dagger}=\left(\bigotimes_{l=1}^{j-1} \sigma_{l}^{z}\right) \frac{\sigma_{j}^{x}-\imath \sigma_{j}^{y}}{2},
$$

the XY model can be brought into a free fermionic form. Before doing so, however, states must be separated according to their parity $\Pi^{z}$, since negative (positive) parity corresponds to (anti-)periodic boundary 
conditions applied to the fermions. Thus, the XY chain Hamiltonian can be written as

$$
H=\frac{1+\Pi^{z}}{2} H^{+} \frac{1+\Pi^{z}}{2}+\frac{1-\Pi^{z}}{2} H^{-} \frac{1-\Pi^{z}}{2},
$$

where the exact expressions of $H^{+}$and $H^{-}$can be found in the supplementary material (https://stacks.iop. org/NJP/22/083024/mmedia). From these Hamiltonians it is possible to determine, following the method described in details in the supplementary data. The fundamental two-point correlation functions. These correlations are expressed as the determinant of a Toeplitz matrix, whose asymptotic behavior can be evaluated analytically [50]:

$$
\begin{aligned}
& \left\langle\sigma_{j}^{x} \sigma_{j+r}^{x}\right\rangle \stackrel{r \rightarrow \infty}{\simeq} \frac{2}{\pi \sqrt{1-\cot ^{2} \phi}} \frac{\cot ^{r} \phi}{r}+\ldots \\
& \left\langle\sigma_{j}^{y} \sigma_{j+r}^{y}\right\rangle \stackrel{r \rightarrow \infty}{\simeq} \begin{cases}\sqrt{1-\cot ^{2} \phi}\left(1+\frac{4}{\pi}\left(\frac{\cot \phi}{1-\cot ^{2} \phi}\right)^{2} \frac{\cot ^{r} \phi}{r^{2}}+\ldots\right) & r=2 m \\
\sqrt{1-\cot ^{2} \phi}\left(1+\frac{2}{\pi}\left(\frac{\cot \phi}{1-\cot ^{2} \phi}\right)^{2} \frac{1+\cot ^{2} \phi}{\cot \phi} \frac{\cot ^{r} \phi}{r^{2}}+\ldots\right) & r=2 m+1\end{cases} \\
& \left\langle\sigma_{j}^{z} \sigma_{j+r}^{z}\right\rangle \stackrel{r \rightarrow \infty}{\simeq} \begin{cases}0 & r=2 m \\
-\frac{2}{\pi} \frac{\cot ^{r} \phi}{r^{2}}+\ldots & r=2 m+1\end{cases}
\end{aligned}
$$

From these large $r$ behavior, taking into account the cluster decomposition hypothesis, we can extract the different magnetizations $m_{\alpha} \equiv\left\langle\sigma_{j}^{\alpha}\right\rangle$

$$
m_{x}=m_{z}=0, \quad m_{y}=\left(1-\cot ^{2} \phi\right)^{1 / 4} .
$$

However, on an odd-length chain at $h=0$, exploiting the symmetries that we have already illustrated, we can provide a direct way to evaluate the different magnetizations even in finite systems. In fact, if $\left|g_{z}\right\rangle$ is one of the degenerate ground states with definite $z$-parity which can be constructed in terms of the Bogoliuobov fermions [44], we can generate a ground state with definite $x$-parity ( $y$-parity) as $\left|g_{x}\right\rangle \equiv \frac{1}{\sqrt{2}}\left(1+\Pi^{x}\right)\left|g_{z}\right\rangle,\left(\left|g_{y}\right\rangle \equiv \frac{1}{\sqrt{2}}\left(1+\Pi^{y}\right)\left|g_{z}\right\rangle\right)$. All these states have a vanishing magnetization in the orthogonal directions while along their own axes we have

$$
\begin{aligned}
& \left\langle g_{x}\left|\sigma_{j}^{x}\right| g_{x}\right\rangle=\left\langle g_{z}\left|\sigma_{j}^{x} \Pi^{x}\right| g_{z}\right\rangle=\left\langle g_{z}\left|\tilde{\Pi}_{j}^{x}\right| g_{z}\right\rangle \\
& \left\langle g_{y}\left|\sigma_{j}^{y}\right| g_{y}\right\rangle=\left\langle g_{z}\left|\sigma_{j}^{y} \Pi^{y}\right| g_{z}\right\rangle=\left\langle g_{z}\left|\tilde{\Pi}_{j}^{y}\right| g_{z}\right\rangle
\end{aligned}
$$

where $\tilde{\Pi}_{j}^{\alpha} \equiv \bigotimes_{l \neq j} \sigma_{l}^{\alpha}$ with $\alpha$ running between $x$ and $y$. These states are the analytical continuation at $h=0$ of the zero-temperature 'thermal' ground state that spontaneously breaks the $\mathbb{Z}_{2}$ symmetry.

Note that in this way, we turn the calculation of the expectation value of an operator defined on a single-spin with respect to a ground state with a mixed $z$-parity into that of a string made by an even number of spin operators on a definite $z$-parity state, which is a standard problem. Thus the rhs of equation (9) can be written again as the determinant of a Toeplitz matrix, whose asymptotic behavior can be studied analytically, similarly to what has been done in [51]. This novel 'trick' can be understood as originating from the fact that, at zero external fields, the chain equation (1) has particle/hole duality and that, on a chain with an odd number of sites, this symmetry relates states with different parities. The result of such analysis reproduces equation (8), proving the consistency of the two methods of evaluation for the order parameters. More details on this direct approach in the supplementary material.

While for $\delta=0$ we can evaluate the magnetizations using the analytical 'trick', for $\delta \neq 0$ we have to resort to numerical solutions. In figure 1 we present some typical results for the finite size magnetizations for the XY and XYZ chain, showing a quick exponential decay in $N$ of $m_{x}$ and $m_{z}$ to zero and a fast saturation of $m_{y}$ (note that each plotted magnetization $m_{\alpha}$ is calculated with respect to the corresponding ground state $\left.\left|g_{\alpha}\right\rangle\right)$.

\section{The frustrated case}

We now turn to the case with $(\phi \in(-\pi / 4,0])$, where the boundary conditions induce topological frustration. The effect of frustration has been recently studied in detail in references $[14,15,52]$. For $\delta=0$, the model can be solved through the same steps used in the traditional cases and exactly mapped into a 

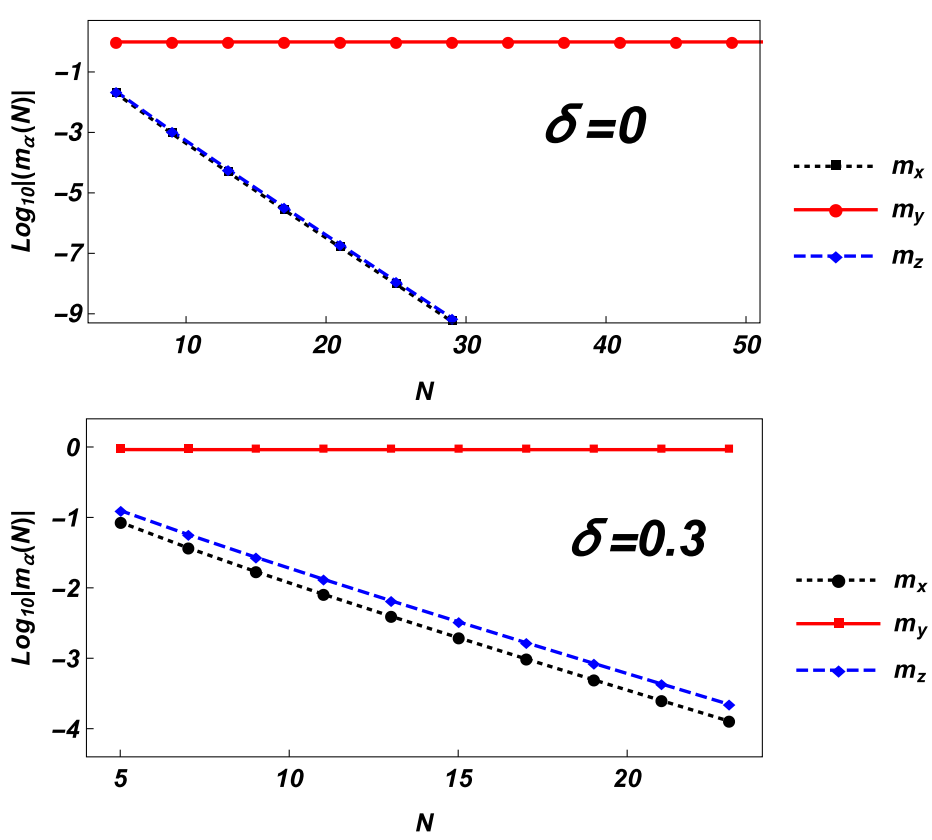

Figure 1. Magnetizations along the three axis (in absolute value) as a function of the chain length for the $y$ FM phase at $\phi=-1.32$. The upper panel dots represent the data gathered settings $\delta=0$ and using the 'trick' discussed in the text to evaluate the magnetizations as determinants of $\frac{N-1}{2} \times \frac{N-1}{2}$ matrices, while the dots in the lower one are obtained taking $\delta=0.3$ and using exact numerical diagonalization. Regardless the value of $\delta, m_{y}$ quickly saturates to its asymptotic finite value, while $m_{x}$ and $m_{z}$ decay to zero exponentially fast, as shown by the best fit lines (plots presented in logarithmic scale).

system of free fermions. In the ferromagnetic phase, the degeneracy between the different parity states is due to the presence of a single negative energy mode (only in one of the parity sectors), whose occupation lowers the energy of those states. With frustrations, the negative energy mode moves into the other parity sector and, because of the parity selection rules in (4), it cannot be excited alone. Therefore, the effect of frustration is that the lowest energy states in each parity sector in (4) are not admissible.

The two degenerate ground states thus carry the signature of a single delocalized excitation and lie at the bottom of a band of states in which this excitation moves with different momenta (with an approximate Galilean dispersion relation). Hence, another effect of frustration is to close the gap that would otherwise exist.

Let us then repeat the extraction of the order parameters in the $x$ AFM phase, following the same procedure we followed for $y$ FM. However, in the present case, the analytical computation of the spin correlations along the $x$ and $y$ directions requires the knowledge of the asymptotic behavior of a new type determinants, whose symbol contains a delta function with a peak at the momentum of the excitation. The details of the analytical computation are given in [53], where such determinants are studied. Here we check these results numerically. The details are given in the supplementary material, where also the correlation along $z$ is computed. The results are

$$
\begin{aligned}
& \left\langle\sigma_{j}^{x} \sigma_{j+r}^{x}\right\rangle \stackrel{r \rightarrow \infty}{\simeq}\left\{\begin{array}{lc}
\sqrt{1-\tan ^{2} \phi}\left(1-\frac{2 r}{N}\right)\left[1+\frac{4}{\pi}\left(\frac{\tan \phi}{1-\tan ^{2} \phi}\right)^{2} \frac{\tan ^{r} \phi}{r^{2}}+\ldots\right] & r=2 m \\
-\sqrt{1-\tan ^{2} \phi}\left(1-\frac{2 r}{N}\right)\left[1+\frac{2}{\pi}\left(\frac{\tan \phi}{1-\tan ^{2} \phi}\right)^{2} \frac{1+\tan ^{2} \phi}{\tan \phi} \frac{\tan ^{r} \phi}{r^{2}}+\ldots\right] & r=2 m+1
\end{array}\right. \\
& \left\langle\sigma_{j}^{y} \sigma_{j+r}^{y}\right\rangle \stackrel{r \rightarrow \infty}{\simeq}\left\{\begin{array}{lc}
\frac{2}{\pi \sqrt{1-\tan ^{2} \phi}} \frac{(-\tan \phi)^{r}}{r}+2^{\frac{5}{2}} \frac{1}{1+\tan \phi} \frac{(-\tan \phi)^{\frac{r}{2}}}{N \sqrt{\pi r}}+\ldots & r=2 m \\
\frac{2}{\pi \sqrt{1-\tan ^{2} \phi}} \frac{(-\tan \phi)^{r}}{r}+2^{\frac{3}{2}} \frac{(-\tan \phi)^{\frac{1}{2}}+(-\tan \phi)^{-\frac{1}{2}}}{1+\tan \phi} \frac{(-\tan \phi)^{\frac{r}{2}}}{N \sqrt{\pi r}}+\ldots & r=2 m+1
\end{array}\right.
\end{aligned}
$$



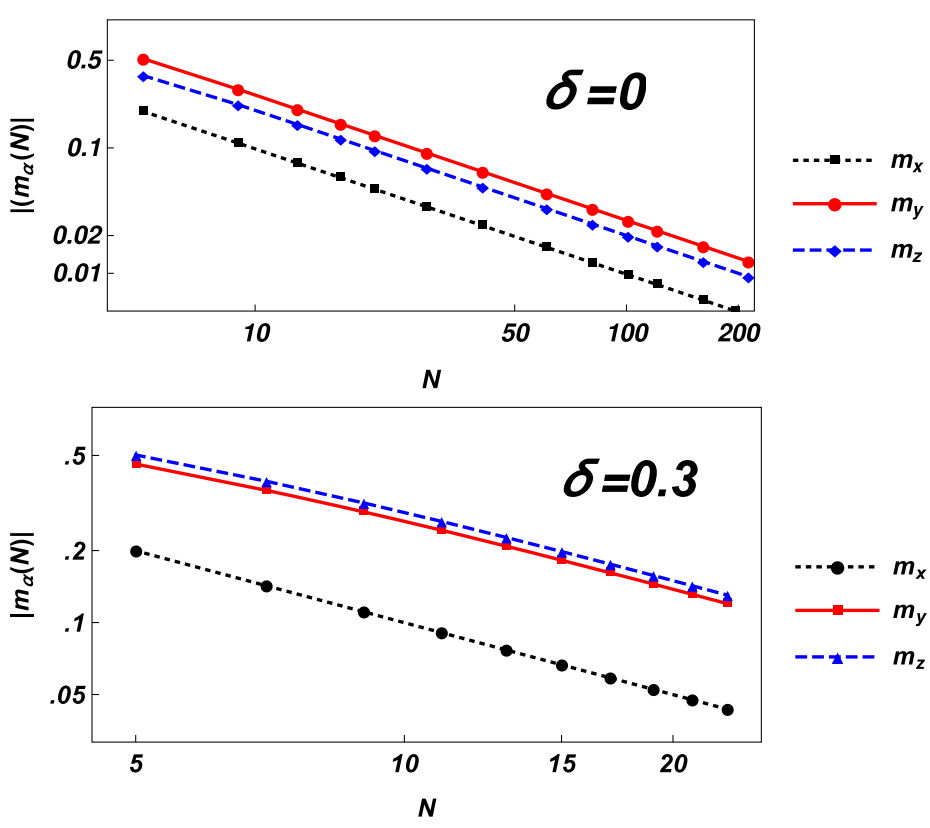

Figure 2. Magnetizations along the three axis (in absolute value) as a function of the chain length for the $x$ AFM/MFM phase at $\phi=-0.25$. The upper panel dots represent the data gathered settings $\delta=0$ using the 'trick' discussed in the text to evaluate the magnetizations as determinants of $\frac{N-1}{2} \times \frac{N-1}{2}$ matrices, while the dots in the lower one are obtained taking $\delta=0.3$ and using exact numerical diagonalization. Regardless of the value of $\delta$, we see how all magnetizations decay just algebraically to zero, as shown by the best fit lines (plots presented in log-log-scale).

$$
\left\langle\sigma _ { j } ^ { z } \sigma _ { j + r } ^ { z } \stackrel { r \rightarrow \infty } { \simeq } ^ { \simeq } \left\{\begin{array}{ll}
0 & r=2 m \\
-\frac{2}{\pi} \frac{\tan ^{r} \phi}{r^{2}}+2^{\frac{3}{2}} \sqrt{1-\tan ^{2} \phi} \frac{(-\tan \phi)^{\frac{r-1}{2}}}{N \sqrt{\pi r}} \ldots & r=2 m+1
\end{array}\right.\right.
$$

While they imply quite clearly that $m_{y}=m_{z}=0$ (in accordance to expectations), the extraction of $m_{x}$ is more subtle: using the standard prescription of taking $N \rightarrow \infty$ first, one would get $m_{x}=\left(1-\tan ^{2} \phi\right)^{1 / 4}$. However, one could argue [15] that a better procedure would be to evaluate equation (10) at antipodal points $r \sim N / 2$ to minimize the correlations and then take the thermodynamic limit. In this way, one would get $m_{x}=\frac{1}{N}\left(1-\tan ^{2} \phi\right)^{1 / 4} \stackrel{N \rightarrow \infty}{\rightarrow} 0$.

It is thus important that we can directly access the single spin magnetization using equation (9). Once more, the expectation values can be cast as determinants of Toeplitz matrices, whose behaviors are depicted in the upper panel of figure 2: all magnetizations are characterized by an algebraic decay to zero with the system size. The analytical results for the XY chain are given in the supplementary material, and demonstrate clearly this property.

Several elements are surprising in these results. The most evident one is that FBC kills the magnetization in the $x$-direction, that on an open or even-length chain would be finite, thus seemingly contradicting the independence of Landau construction from boundary conditions. Note that a finite spontaneous magnetization can be measured in any finite system, although it decreases algebraically with the system size, a phenomenon we term 'mesoscopic magnetization'. Quite surprisingly, however, this finite-size spontaneous magnetization is not staggered, but rather ferromagnetic-looking (thus, we will call the AFM phase with FBC, a mesoscopic ferromagnetic phase, MFM). In hindsight, we could have expected this, since a staggered magnetization would have not been compatible with $\mathrm{PBC}$ with an odd number of sites (note that this is not a problem for the 2-point function, since its range naturally does not extend beyond one periodicity).

This analytical outcomes are corroborated by exact numerical diagonalization results, which allow us to extended our analysis to the XYZ $(\delta \neq 0)$ ring, (see the lower panel of figure 2$)$. In figure 3 we plot the behavior of the magnetizations as a function of $\phi$ for $\delta=0.3$ for several chain lengths $N$ : while in the $y \mathrm{FM}$ phase there is little dependence on $N$ as the saturation values are reached quickly, in the MFM phase we observe the slow, algebraic decay toward zero of the order parameters.

It is rather surprising that a finite chain, unable to sustain AFM order, would nonetheless generate a ferromagnetic spontaneous magnetization and that in any finite system, a phase with a dominant interaction along the $x$ direction would show the weakest spontaneous magnetization in that direction, with 

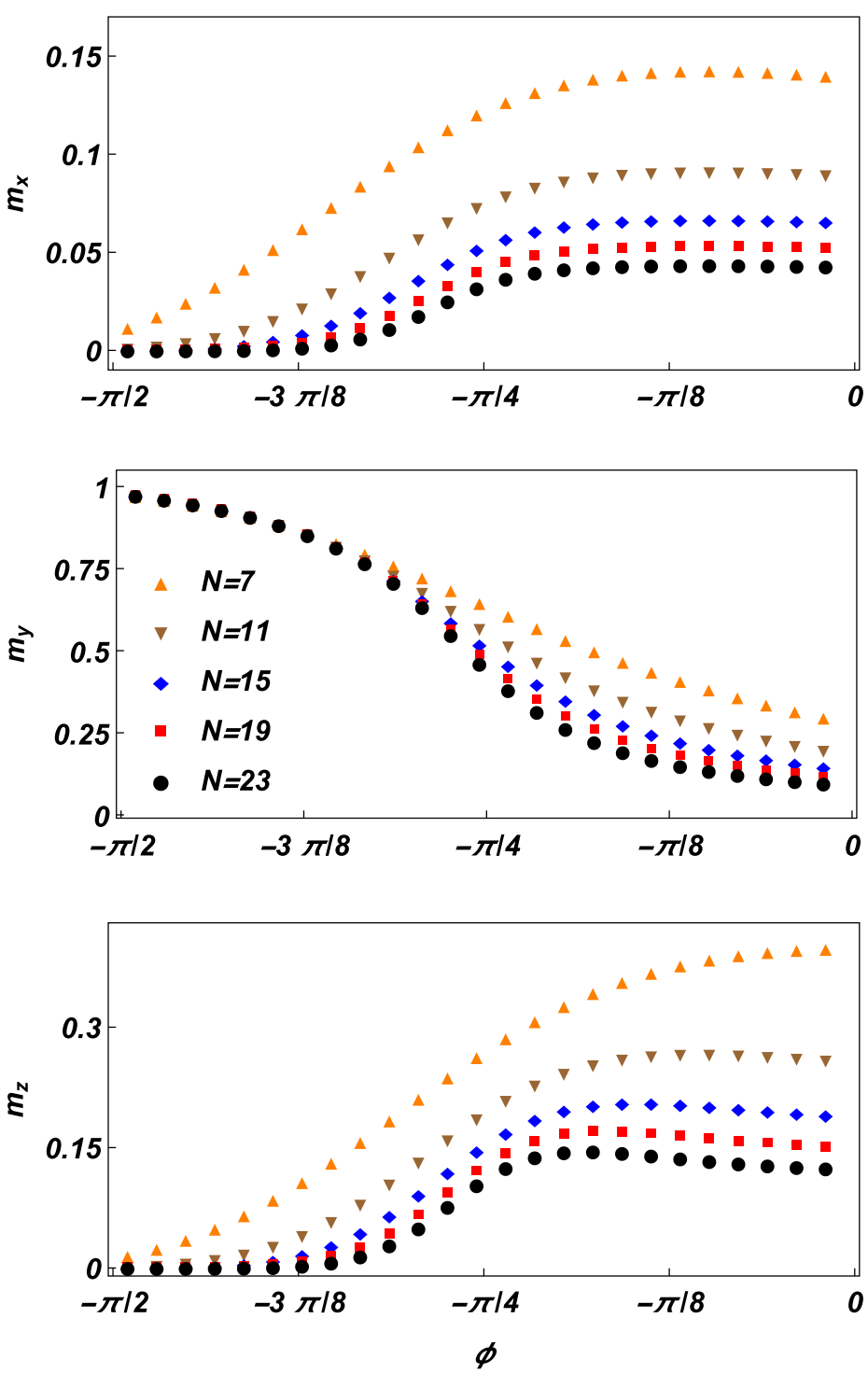

Figure 3. Plot of the magnetizations as a function of $\phi$ for $\delta=0.3$ for several system sizes. The $y$ FM phase $(\phi<-\pi / 4)$ shows a fast approach to saturation, while for the frustrated case the decay toward zero is algebraically slow.

$m_{y}$ being the strongest one (once more, these magnetization refers to different states $\left|g_{\alpha}\right\rangle$ ). Finally, we remark that FBC also seems to somewhat spoil the cluster decomposition, since the non-staggered mesoscopic magnetization we find is not compatible with (10), although both of them vanish in the thermodynamic limit.

\section{Conclusions}

We have presented a comparative study of the ferromagnetic and AFM frustrated case for a XYZ chain, showing that, contrary to expectations, the boundary conditions are able to destroy local order. We have done so, by realizing that, with no external field, we can exploit particle/hole duality to construct an exact ground state at finite sizes that break the $\mathbb{Z}_{2}$ symmetry. For the XY chain, we can express the one-point function as the determinant of a Toeplitz matrix and evaluate it analytically, while for the interacting cases we can numerically diagonalize the model and calculate the expectation values. We benchmarked these procedures on a ferromagnetic phase with FBC to show that they reproduce the expected results equation (8), while in an AFM phase the magnetizations, while finite in a finite chain, decay toward zero algebraically in the thermodynamic limit. Furthermore, despite a dominant AFM interaction, no magnetization shows a staggered behavior: we thus term this pseudo-phase generated by FBC a mesoscopic ferromagnetic phase (MFM).

While we worked at zero fields to have an exact degeneracy on any finite chain and thus to have perfect control of finite size effects, we expect our results to remain valid also with a finite external field (at least up 
to some threshold). While the exact degeneracy is lifted by a finite field, the energy difference between the lowest energy states in the two parity sectors closes in the thermodynamic limit. However, while this closing is usually exponentially fast, with frustration is only algebraic and accompanied by a similar behavior with respect of other low energy states $[14,15]$. This different behavior is mirrored by the two-point function (10), which can be extended with a qualitative difference for finite $h$ and indicates a vanishing order parameter when evaluated at antipodal points [15]. The extension of our analysis to finite $h$ will be the subject of future work, but the existence of a MFM phase would be experimentally detectable, with signatures like those in figure 3 easily measurable. The reason for which it has not been seen until now lies in the (surely not extreme) difficulty in realizing a ring geometry and in the expectation that every boundary condition would yield the same result, an expectation that our work put into question.

Nonetheless, we should remark that our results are fully consistent with a straightforward perturbative calculation starting from the classical frustrated Ising chain, as we show in the supplementary material. Our original contribution is to have found an exact way to approach the thermodynamic limit and to calculate the order parameter. Moreover, with our techniques, we can prove that our result is resilient against a coupling defect breaking translational invariance [54], which further ensures the experimental observability of our claim.

Our results are surprising because they seemingly contradict the assumption that boundary conditions cannot influence the bulk behavior of a system and therefore certainly not destroy local order. We do not know at the moment how to reconcile this apparent paradox and we invite the community to help us in looking for a general explanation. For the moment we can contribute with a couple of observations. The first is that FBC provides a non-local contribution to the system since frustration arises from an incompatibility between local and global order. Thus, it is possible that the problem we consider can have a topological origin that defies the Landau paradigm. Another, somewhat more technical angle, is that in our class of models, the single spin magnetization is dual to a non-local correlator, see equation (9). From this point of view, it is not surprising that a non-local function is sensitive to the boundary conditions. Nonetheless, we have to admit that it seems to us rather paradoxical to consider single-site magnetizations as non-local quantities.

\section{Acknowledgments}

We acknowledge support from the European Regional Development Fund-the Competitiveness and Cohesion Operational Programme (KK.01.1.1.06-RBI TWIN SIN). The work is also partially supported by the Croatian Science Fund Project No. IP-2016-6-3347. SMG and FF also acknowledge support from the QuantiXLie Center of Excellence, a project co-financed by the Croatian Government and European Union through the European Regional Development Fund-the Competitiveness and Cohesion Operational Programme (Grant KK.01.1.1.01.0004) and Croatian Science Fund Project No. IP-2019-4-3321.

\section{ORCID iDs}

Fabio Franchini (D) https://orcid.org/0000-0002-3429-8189

\section{References}

[1] Landau L D, Lifshitz E M and Pitaevskij L P 1978 Statistical Physics (Oxford: Pergamon)

[2] Chandler D 1987 Introduction to Modern Statistical Mechanics 1st edn (Oxford: Oxford University Press)

[3] Anderson P W 1997 Basic Notions of Condensed Matter Physics (Reading, MA: Addison-Wesley)

[4] Coleman P 2016 Introduction to Many-Body Physics (Cambridge: Cambridge University Press)

[5] Stone M (ed) 1992 Quantum Hall Effect (Singapore: World Scientific)

[6] Wen X-G 2004 Quantum Field Theory of Many-body Systems: From the Origin of Sound to an Origin of Light and Electrons (Oxford: Oxford University Press)

[7] Nayak C, Simon S H, Stern A, Freedman M and Das Sarma S 2008 Rev. Mod. Phys. 801083

[8] Hasan M Z and Kane C L 2010 Rev. Mod. Phys. 823045

[9] Fradkin E 2013 Field Theories of Condensed Matter Physics (Cambridge: Cambridge University Press)

[10] Bernevig B A and Hughes T L 2013 Topological Insulators and Topological Superconductors (Princeton, NJ: Princeton University Press)

[11] Giampaolo S M and Hiesmayr B C 2015 Phys. Rev. A 92012306

[12] Witten E 2016 Rev. Mod. Phys. 8835001

[13] Zeng B, Chen X, Zhou D-L and Wen X-G 2019 Quantum Information Meets Quantum Matter: From Quantum Entanglement to Topological Phases of Many-Body Systems (Berlin: Springer)

[14] Dong J-J, Li P and Chen Q-H 2016 J. Stat. Mech. 113102 
[15] Giampaolo S M, Ramos F B and Franchini F 2019 J. Phys. Commun. 3081001

[16] Affleck I 1995 Acta Phys. Pol. B 261869

[17] Durganandini P 1996 Phys. Rev. B 53 R8832

[18] Cardy J 1984 Nucl. Phys. B 2404

[19] Cardy J 2004 arXiv:hep-th/0411189

[20] Di Francesco P, Mathieu P and Senechal D 1999 Conformal Field Theory (Berlin: Springer)

[21] Korepin V E, Bogoliubov N M and Izergin A G 1997 Quantum Inverse Scattering Method and Correlation Functions (Cambridge: Cambridge University Press)

[22] Korepin V E and Zinn-Justin P 2000 J. Phys. A 337053

[23] Zinn-Justin P 2002 arXiv:cond-mat/0205192

[24] Colomo F and Pronko A G 2010 J. Stat. Phys. 138662

[25] Bleher P and Liechty K 2013 Random Matrices and the Six-Vertex Model (CRM Monographs Series vol 32) (Providence RI: American Mathematical Society)

[26] Colomo F and Sportiello A 2016 J. Stat. Phys. 1641488

[27] Allegra N, Dubail J, Stéphan J-M and Viti J 2016 J. Stat. Mech. 053108

[28] Reshetikhin N and Sridhar A 2017 Commun. Math. Phys. 356535

[29] Di Francesco P and Guitter E 2018 J. Phys. A: Math. Theor. 51355201

[30] Colomo F, Pronko A G and Sportiello A 2018 J. Stat. Phys. 1741

[31] Toulouse G 1977 Commun. Phys. 2115

[32] Vannimenus J and Toulouse G 1977 J. Phys. C 10 L537

[33] Wolf M M, Verstraete F and Cirac J I 2003 Int. J. Quantum Inf. 1465

[34] Giampaolo S M, Gualdi G, Monras A and Illuminati F 2011 Phys. Rev. Lett. 107260602

[35] Marzolino U, Giampaolo S M and Illuminati F 2013 Phys. Rev. A 88020301

[36] Sadoc J F and Mosseri R 2007 Geometrical Frustration (Cambridge: Cambridge University Press)

[37] Lacroix C, Mendels P and Mila F (ed) 2011 Introduction to Frustrated Magnetism: Materials, Experiments, Theory (Solid-State Sciences vol 164) (Berlin: Springer)

[38] Diep H T 2013 Frustrated Spin Systems (Singapore: World Scientific)

[39] Wannier G H 1950 Phys. Rev. 79357

[40] Burkhardt T W and Guim I 1985 J. Phys. A: Math. Gen. 18 L33

[41] Cabrera G G and Jullien R 1987 Phys. Rev. B 357062

[42] Campostrini M, Pelissetto A and Vicari E 2015 Phys. Rev. E 91042123

[43] Ercolessi E, Evangelisti S, Franchini F and Ravanini F 2013 Phys. Rev. B 88104418

[44] Franchini F 2017 An Introduction to Integrable Techniques For One-Dimensional Quantum Systems (Lecture Notes in Physics vol 940) (Berlin: Springer)

[45] Marić V, Giampaolo S M, Kuić D and Franchini F 2020 arXiv:2002.07197

[46] Sachdev S 2011 Quantum Phase Transitions (Cambridge: Cambridge University Press)

[47] Damski B and Rams M M 2014 J. Phys. A 47025303

[48] Lieb E, Schultz T and Mattis D 1961 Ann. Phys. 16 407-66

[49] Jordan P and Wigner E 1928 Z. Phys. 47631

[50] McCoy B M 1968 Phys. Rev. 173531

[51] Barouch E and McCoy B M 1971 Phys. Rev. A 3786

[52] Dong J-J and Li P 2017 Mod. Phys. Lett. B 311750061

[53] Marić V and Franchini F 2020 arXiv:2006.01922

[54] Torre G, Marić V, Franchini F and Giampaolo S M in preparation 